\title{
ANÁlise da ASSOCIAÇÃo eNTRE SANEAMENTO E SAÚde NOS ESTADOS BRASILEIROS, EMPREGANDO DADOS SECUNDÁRIOS DO BANCO DE DADOS INDICADORES E DADOS BÁSICOS PARA A SAÚDE 2003- IDB 2003
}

\author{
ANALYSIS OF ASSOCIATION BETWEEN SANITATION AND HEALTH IN BRAZILIAN \\ STATES, USING SECONDARY DATA FROM DATA BANK INDICADORES E DADOS \\ BÁSICOS PARA A SAÚDE 2003 - IDB 2003
}

\section{JÚLIO CÉSAR TEIXEIRA}

Engenheiro Civil e de Segurança no Trabalho. Doutor em Saneamento, Meio Ambiente e Recursos Hídricos. Professor Adjunto do Departamento de Hidráulica e Saneamento da Universidade Federal de Juiz de Fora

\author{
RENATA LOPES GUILHERMINO \\ Engenheiranda Civil pela Universidade Federal de Juiz de Fora
}

Recebido: 23/06/05 Aceito: 03/08/06

\section{RESUMO}

O objetivo do presente trabalho foi avaliar a associação entre condiçōes de saneamento - cobertura populacional por redes de abastecimento de água, por sistemas de esgotamento sanitário e por serviços de coleta de lixo - e indicadores epidemiológicos - taxa de mortalidade infantil, mortalidade proporcional por doença diarréica aguda em menores de cinco anos de idade e mortalidade proporcional por doenças infecciosas e parasitárias para todas as idades - nos estados brasileiros, utilizando dados secundários do banco de dados Indicadores e Dados Básicos para a Saúde 2003 - IDB 2003 - do Ministério da Saúde.

PALAVRAS CHAVE: Associação, saneamento, saúde, estados brasileiros, IDB 2003.

\begin{abstract}
The objective of the present study was to evaluate the association between sanitation and epidemiological indicators - in the brazilian states. Secondary data from data bank Indicadores $e$ Dados Básicos para a Saúde 2003 - IDB 2003 - from Ministry of Health were used. Three types of sanitation were compared: population coverage by water supply system, by sewage disposal, and by domestic garbage collection services. They were compared with infant mortality rate, proportional mortality by acute diarrhea among infants under five years of age, and proportional mortality by infectious and parasitic diseases for all ages.
\end{abstract}

KEYWORDS: Association, sanitation, health, brazilian states, IDB 2003.

\section{INTRODUÇÃO}

O reconhecimento da importância do saneamento e de sua associação com a saúde humana remonta às mais antigas culturas. Ruínas de uma grande civilização ao norte da Índia, com mais de 4.000 anos de idade, indicam evidências da existência de hábitos higiênicos, incluindo a presença de banheiros e de sistemas de coleta de esgotos sanitários nas edificaçôes, além de drenagem nos arruamentos. Outros povos, como os egípcios, dispunham de sistemas de drenagem de água, além da existência de grandes aquedutos. Também destacamse os cuidados com o destino dos dejetos na cultura creto-micênica e as noçôes de engenharia sanitária dos quíchuas (Rosen, 1994).

Segundo Heller (1997), o avanço das práticas sanitárias coletivas encontrou sua expressão mais marcante na Antigüidade nos aquedutos, banhos públicos, termas e esgotos da Roma antiga, tendo como símbolo histórico máximo a Cloaca Máxima de Roma.

$\mathrm{Na}$ trajetória mais recente da saúde pública, Jonh Snow (1990), em sua histórica pesquisa concluída em 1854, já comprovava cientificamente a associação entre a água contaminada por esgotos sanitários consumida pela população de Londres e a incidência de cólera. A investigação de Snow ocorreu cerca de 20 anos antes do início da era bacteriológica, com Pasteur, Koch e outros colaboradores (Rosen, 1994).

O processo de implantação de sistemas coletivos de saneamento, iniciado nos fins do século XIX e início do século XX, apontou para uma melhoria constante do estado de saúde das populaçōes beneficiadas, independente da existência de evidências científicas, no início do século $\mathrm{XX}$, que permitissem associar melhorias na saúde pública à implantação de sistemas coletivos de saneamento.

Hoje, sabe-se que os serviços de saneamento são de vital importância para proteger a saúde da população, minimizar as conseqüências da pobreza e proteger o meio ambiente. No entanto, 
os recursos financeiros disponíveis para o setor são escassos no Brasil, a despeito das carências observadas. Logo, a ausência de estudos sobre a influência da cobertura populacional por serviços de saneamento sobre as condiçōes de saúde existentes nas diferentes unidades da federação constitui uma importante lacuna nas pesquisas no campo do saneamento no Brasil.

Os países em desenvolvimento, entre os quais o Brasil, entraram no terceiro milênio ressuscitando patologias do início do século XX. A ausência de serviços de saneamento tem resultado em precárias condições de saúde de uma parte significativa da população brasileira, com a incidência de doenças, destacando-se as de veiculação hídrica, tais como diarréias, hepatite, cólera, parasitoses intestinais, febre tifóide, entre outras. Em conseqüência, a taxa de mortalidade infantil no Brasil ainda é elevada - 27 óbitos de menores de um ano por mil nascidos vivos, mas sua queda é inegável: em 1970 ela estava próxima de 100 por mil nascidos (IBGE, 2005).

Confirmando a afirmativa anterior, segundo a Conferência das Nações Unidas sobre Meio Ambiente e Desenvolvimento (1997), na maioria dos países em desenvolvimento, a impropriedade e a carência de infra-estrutura sanitária é responsável pela alta morbidade por doenças de veiculação hídrica e por um grande número de mortes evitáveis a cada ano. Nesses países, verificam-se condições que tendem a piorar devido às necessidades crescentes de serviços e ações de saneamento ambiental, que excedem a capacidade dos governos de reagir adequadamente.

No Brasil, de acordo com a publicação "Indicadores e Dados Básicos para a Saúde 2003” (IDB, 2004), ainda há muita carência de serviços de saneamento básico, como indicam os dados abaixo:

- $80 \%$ da população contam com abastecimento de água por rede geral;

- 66\% da população dispóem de esgotamento sanitário por rede geral ou fossa; coletado.

-83\% da população têm seu lixo

No entanto, na última década do século XX e na primeira década do século XXI, cresceu a compreensão que as atividades de saneamento integram as ações de saúde pública, uma vez que visam a saúde da população, no seu sentido mais amplo de promoção e proteção da saúde coletiva.

Ainda, deve ser destacado o papel crescente da educação ambiental nos programas de saneamento, uma vez que, sem a participação da população, dificilmente os programas de saneamento obtêm êxito.

Quanto às populações não atendidas por serviços de saneamento adequados, Silva \& Alves (1999) sustentam que, no Brasil, estas se concentram nas periferias das grandes e médias cidades - nos chamados assentamentos subnormais -, nos pequenos aglomerados urbanos - cidades com menos de 20.000 habitantes - e nas regiões mais pobres do país - com o destaque para as regiōes Norte e Nordeste do Brasil.

"As enfermidades associadas à deficiência ou inexistência de saneamento ambiental e a conseqüente melhoria da saúde devido à implantação de tais medidas têm sido objeto de discussão em diversos estudos" (Moraes, 1997).

Paes \& Silva (1999), por exemplo, destacam a relevância do papel das doenças infecciosas e parasitárias entre as causas de morte no Brasil, sustentando que estas estão diretamente associadas a condiçốes de habitação e de higiene precárias.

Ainda sobre precárias condições de saneamento, Benicio \& Monteiro (2000) afirmam ser estas um fator determinante para a alta incidência da diarréia infantil no país, tida também como uma das principais causas da mortalidade infantil.

É sabido que benefícios específicos de intervençōes de saneamento ambiental incluem a diminuição da morbidade devido às doenças diarréicas e parasitárias e à melhoria do estado nutricional das crianças (Teixeira, 2003).

Contudo, a avaliação dos efeitos das medidas de saneamento ambiental sobre a morbi-mortalidade infantil é de difícil realização e os resultados dependem de um número considerável de outros indicadores como, por exemplo, demográficos, sócio-econômicos, de fatores de risco, de gastos públicos e de cobertura por serviços de saúde, para sua interpretação adequada. Deve-se, então, quando da realização desses estudos, levar em consideração várias questôes metodológicas para que não venham a invalidar os resultados (Blum \& Feachem, 1983).

Resultados do Censo 2000 revelaram o inchaço da periferia das cidades, com índices crescentes de favelização, desemprego e miséria. É nesse contexto que o presente trabalho terá como objetivo avaliar o impacto da cobertura dos sistemas de saneamento sobre a saúde das populaçôes residentes nos diferentes estados da federação, de modo a contribuir para a melhoria da qualidade de vida da população brasileira, bem como para o desenvolvimento de políticas públicas de saneamento e saúde.

A hipótese submetida à teste neste trabalho foi:

"A ampliação de infra-estrutura sanitária em estados com precárias condições de saneamento ambiental é um investimento capaz de melhorar a qualidade de vida da população residente nestes estados, por meio da melhoria de sua saúde e da redução de gastos com medicina curativa".

O presente trabalho apresenta alguns aspectos de originalidade, como apreendido de revisão de literatura especializada, a saber:

- compreender a relação entre saneamento e saúde nos estados brasileiros, a partir de dados secundários constantes da publicação anual Indicadores e Dados Básicos para a Saúde, do Ministério da Saúde;

- avaliar o método ecológico no estudo desta relação.

\section{OBJETIVO}

Avaliar a associação entre condições de saneamento - cobertura populacional por redes de abastecimento de água, por sistemas de esgotamento sanitário e por serviços de coleta de lixo - e indicadores epidemiológicos - taxa de mortalidade infantil, mortalidade proporcional por doença diarréica aguda em menores de cinco anos de idade e mortalidade proporcional por doenças infecciosas e parasitárias para todas as idades - nos estados brasileiros, utilizando dados secundários do banco de dados Indicadores e Dados Básicos para a Saúde 2003 - IDB 2003 - do Ministério da Saúde (IDB, 2004).

\section{METODOLOGIA}

\section{Áreas geográficas abrangidas}

O universo da pesquisa foi composto pelos 27 estados e pelo distrito federal integrantes da República Federativa do Brasil no ano de 2000, com 
população de 169.799.170 habitantes, distribuída por 44.795.101 domicílios (IBGE, 2002).

\section{Delineamento epidemiológico}

O método epidemiológico empregado foi um estudo ecológico, que pode ser melhor compreendido como o estudo epidemiológico que utiliza agregados populacionais como unidade de análise.

\section{Base de informações}

A base de informações foi composta por dados secundários provenientes da publicação Indicadores e Dados Básicos para a Saúde 2003 (IDB, 2004), editado pela Rede Interagencial de Informaçôes para a Saúde - RIPSA, publicado em junho de 2004.

\section{Fatores de risco estudados}

Cada um dos três indicadores de saúde estudados foi analisado por meio de sua correlação com vários outros indicadores, divididos em cinco classes, a saber:

\section{Indicadores demográficos}

- Taxa de crescimento da população (\% de incremento 2001/2002);

- Grau de urbanização, 2002 (\% da população urbana);

- Taxa de fecundidade total, 2001 ( $\mathrm{n}^{\mathrm{o}}$ médio de nascidos vivos por mulher);

- Taxa bruta padronizada de natalidade, 2001 ( $\mathrm{n}^{\mathrm{o}}$ de nascidos vivos por 1.000 hab.);

- Esperança de vida ao nascer, 2002 ( $\mathrm{n}^{\mathrm{o}}$ de anos de vida esperados).

\section{Indicadores sócio-econômicos:}

- Taxa de analfabetismo, 2002 (\% na população de 15 anos e mais de idade);

- Produto Interno Bruto (PIB) per capita, 2001 (R\$/hab.);

- Razão de renda, 2002 ( $n^{\circ}$ de vezes que a renda dos $20 \%$ mais ricos supera a dos $20 \%$ mais pobres em um estado);

- Proporção de pobres, 2002 (\% da população com renda familiar per capita de até meio salário mínimo);

- Taxa de desemprego, 2002 (\% da população economicamente ativa que se encontra sem trabalho).
Indicadores de fatores de risco

- Proporção de nascidos vivos de baixo peso ao nascer, 2001 (\% de nascidos com peso inferior a $2.500 \mathrm{~g}$ );

- Prevalência de déficit ponderal para a idade em crianças menores de 5 anos de idade, 1996 (\%);

- Prevalência de aleitamento materno exclusivo, 1999 (\%);

Indicadores de gastos públicos:

- Gasto público com saúde, como proporção do PIB, 2001 (\%PIB);

- Gasto público com saneamento, como proporção do PIB, 1996 corrigido para 2001 (\%PIB);

Indicadores de cobertura por serviços de saúde e de saneamento:

- Número de consultas médicas SUS por habitante, 2002 (consultas/habitante);

- Número de internações hospitalares SUS por habitante, 2002 (internaçōes/100 habitantes);

- Proporção de gestantes com acompanhamento pré-natal, 2001 (\% com 6 e mais consultas);

- Cobertura vacinal no primeiro ano de vida, 2002 (\% de menores de um ano imunizados);

- Cobertura por redes de abastecimento de água, 2002 (\% da população urbana);

- Cobertura por sistemas de esgotamento sanitário, 2002 (\% da população urbana);

- Cobertura por serviços de coleta de lixo, 2002 (\% da população urbana).

\section{Protocolo adotado para a coleta de dados}

O protocolo utilizado para a coleta de dados teve uma estrutura básica que compreendeu as seguintes seçóes:

- unidade da federação;

- taxa de mortalidade infantil, 2001 ( $\mathrm{n}^{\circ}$ de óbitos infantis por 1.000 nascidos vivos);

- mortalidade proporcional por doença diarréica aguda em menores de cinco anos de idade, 2001 (\% de óbitos informados);

- mortalidade proporcional por doenças infecciosas e parasitárias para todas as idades, 2001(\% de óbitos com causa definida);

- indicadores demográficos, sócioeconômicos, de fatores de risco, de gas- tos públicos e de cobertura por serviços de saúde e de saneamento.

\section{Análise de dados}

A análise epidemiológica foi desenvolvida segundo um processo evolutivo, em etapas, de tal forma a se permitir a determinação progressiva das exposições efetivamente associadas aos indicadores de saúde estudados. Tal processo envolveu, em seqüência, as seguintes atividades:

- análise descritiva dos indicadores para o conjunto das unidades da federação;

- análise de regressão linear simples entre os indicadores epidemiológicos versus indicadores demográficos, sócio-econômicos, de fatores de risco, de gastos públicos, de cobertura por serviços de saúde e de saneamento, "um a um", de modo a avaliar as relaçóes entre os indicadores epidemiológicos - variáveis dependentes - e os demais indicadores - variáveis independentes - e pré-selecionar os indicadores a serem utilizados na regressão linear multivariada em nível de $15 \%$ de significância $(\mathrm{p} \leq 0,15)$;

- análise de regressão linear multivariada onde se procurou identificar as variáveis independentes efetivamente associadas a cada indicador epidemiológico estudado em nível de $5 \%$ de significância $(\mathrm{p} \leq 0,05)$.

Foi utilizado o pacote estatístico SPSS 10.0 - Statistical Package for Social Sciences. Espera-se que tal sistemática tenha sido adequada quanto à sua consistência e ao seu rigor científico.

\section{RESULTADOS}

\section{Taxa de mortalidade infantil}

Da regressão linear simples, vários indicadores foram selecionados pelo critério estabelecido na metodologia, ou seja, selecionar variáveis com valor de $\mathrm{p}$ - significância estatística - igual ou inferior a 0,15 . Apresentaram significância estatística nesta etapa, os seguintes indicadores em ordem de importância da estatística F: proporção de pobres, taxa de analfabetismo, esperança de vida ao nascer, prevalência de déficit ponderal para a idade em crianças menores de 5 anos de idade, PIB per capita, grau de urbanizaçáo, proporção de nascidos vivos de baixo peso ao nascer, cobertura por sistemas 
de esgotamento sanitário, proporção de gestantes com acompanhamento prénatal, cobertura por serviços de coleta de lixo, gasto público com saúde como proporção do $\mathrm{PIB}$, taxa de crescimento da população, taxa bruta de natalidade e taxa de fecundidade total.

Ao se processar a análise de regressão linear múltipla, Tabela 1 , encontrouse um coeficiente $\mathrm{R}^{2}$ ajustado de 0,782 , sendo que as variáveis que permaneceram no modelo final foram: esperança de vida ao nascer $(p<0,001)$ e cobertura por sistemas de esgotamento sanitário $(\mathrm{p}=0,040)$.

Os indicadores esperança de vida ao nascer e cobertura por sistemas de esgotamento sanitário apresentaram coeficiente $\beta$ negativo, mostrando uma relação inversamente proporcional com a taxa de mortalidade infantil.

$\mathrm{Na}$ Figura 1, fica claro que quanto maior a cobertura populacional por serviços adequados de esgotamento sanitário em um estado brasileiro, menor é a mortalidade infantil naquela unidade da federação.

\section{Mortalidade proporcional por doença diarréica aguda em menores de cinco anos de idade}

Da etapa inicial, regressão linear simples, vários indicadores foram selecionados pelo critério estabelecido, ou seja, selecionar variáveis com valor de $\mathrm{p}$ igual ou inferior a 0,15 . Apresentaram significância estatística nesta etapa, os seguintes indicadores em ordem decrescente da estatística F: proporção de pobres, taxa de analfabetismo, PIB per capita, cobertura por sistemas de esgotamento sanitário, esperança de vida ao nascer, proporção de nascidos vivos de baixo peso ao nascer, prevalência de déficit ponderal para a idade em crianças menores de cinco anos de idade, taxa bruta de natalidade, taxa de fecundidade total, grau de urbanização, gasto público com saúde como proporção do PIB, cobertura por serviços de coleta de lixo, proporção de gestantes com acompanhamento pré-natal e cobertura vacinal no primeiro ano de vida.

$\mathrm{Na}$ regressão múltipla, Tabela 2 , encontrou-se um coeficiente $\mathrm{R}^{2}$ de 0,595 , sendo que as variáveis que permaneceram no modelo final foram: cobertura por sistemas de esgotamento sanitário $(p=0,035)$ e proporção de pobres $(\mathrm{p}=0,001)$.

Tabela I - Regressão multivariada linear entre a taxa de mortalidade infantil e indicadores demográficos, sócio-econômicos, fatores de risco, gastos públicos e de cobertura por serviços de saúde e de saneamento

\begin{tabular}{cclccc}
\hline $\mathrm{R}^{2}$ & $\mathrm{R}$ & Variáveis que permaneceram & Coef. $\beta$ & P Valor & Sinal \\
\hline 0,782 & 0,884 & $\begin{array}{l}\text { Esperança de vida ao nascer } \\
\text { Cobertura por sistemas de }\end{array}$ & $-0,791$ & $<0,001$ & - \\
& & & $-0,214$ & 0,040 & - \\
\hline
\end{tabular}

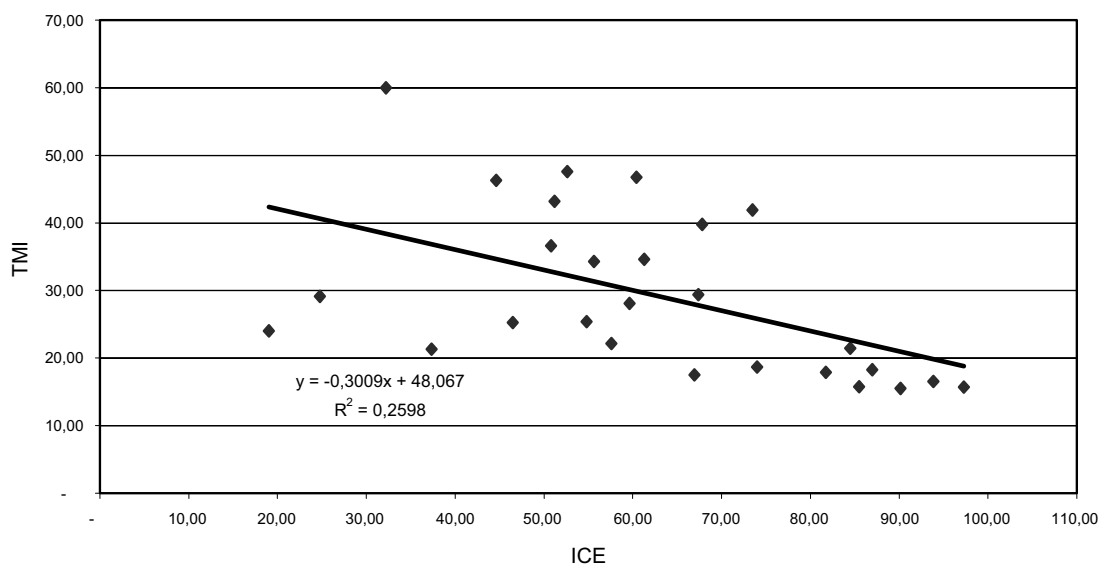

Figura I - Regressão linear simples entre a taxa de mortalidade infantil ( $n^{\circ}$ de óbitos infantis por 1.000 nascidos vivos) e a cobertura por sistemas de esgotamento sanitário (\% da população urbana)

O indicador cobertura por sistemas de esgotamento sanitário apresentou coeficiente $\beta$ negativo, mostrando uma relação inversamente proporcional com a mortalidade por diarréia aguda. Já a proporção de pobres apresentou um coeficiente $\beta$ positivo, demonstrando uma relação diretamente proporcional com este tipo de mortalidade.

$\mathrm{Na}$ Figura 2 observa-se que quanto maior a cobertura populacional por sistemas de esgotamento sanitário em um estado brasileiro, menor é a mortalidade proporcional por doença diarréica aguda em menores de cinco anos de idade em uma unidade da federação

\section{Mortalidade proporcional por doenças infecciosas e parasitárias para todas as idades}

Apresentaram significância estatística na regressão linear simples $(\mathrm{p} \leq 0,15)$, os seguintes indicadores em ordem de significância da estatística F: taxa bruta de natalidade, taxa de fecundidade total, proporção de gestantes com acompanhamento prénatal, proporção de pobres, prevalência de déficit ponderal para a idade em crianças menores de 5 anos de idade, cobertura por redes de abastecimento de água, número de consultas médicas SUS por habitante, cobertura por serviços de coleta de lixo, PIB per capita, proporção de nascidos vivos de baixo peso ao nascer, grau de urbanização, gasto público com saúde como proporção do PIB, esperança de vida ao nascer, cobertura por sistemas de esgotamento sanitário e taxa de analfabetismo.

$\mathrm{Na}$ análise de regressão multivariada, Tabela 3, encontrou-se um coeficiente $\mathrm{R}^{2}$ ajustado de 0,544 , sendo que as variáveis que permaneceram no modelo final foram: cobertura por redes de abastecimento de água $(\mathrm{p}=0,001) \mathrm{e}$ proporção de pobres $(\mathrm{p}=0,001)$.

$\mathrm{O}$ indicador proporção de pobres apresentou coeficiente $\beta$ positivo, mostrando uma relação diretamente proporcional com a mortalidade proporcional por doenças infecciosas e parasitárias para todas as idades. Por outro lado, o indicador cobertura por redes de abastecimento de água apresentou um valor de $\beta$ negativo, mostrando uma relação inversamente proporcional com o indicador epidemiológico em estudo. Os achados são coerentes com os registros da literatura.

Na Figura 3, há evidências de que quanto maior a cobertura populacional por redes de abastecimento de água em um estado brasileiro, menor é a 
mortalidade proporcional por doenças infecciosas e parasitárias para todas as idades naquele estado.

\section{DISCUSSÃO}

Como visto anteriormente, as condiçôes ambientais desempenham papel importante na determinação das condições de vida, principalmente na proteção à saúde, que resulta dos serviços de esgotamento sanitário, aporte adequado de água e coleta de lixo (Tavares \& Monteiro, 1994).

A despeito deste conhecimento, neste trabalho verificou-se, por meio de um estudo ecológico, que a mortalidade infantil, a mortalidade proporcional por doença diarréica aguda em menores de cinco anos de idade e a mortalidade proporcional por doenças infecciosas e parasitárias para todas as idades, nos estados brasileiros, ainda encontram-se associadas com a inexistência ou precariedade dos serviços de saneamento.

Definida pelo Estatuto do Idoso como aquela que tem 60 anos ou mais de idade, a população idosa tem obtido um crescimento absoluto e relativo ao longo dos anos. Esse crescimento é conseqüência da queda nas taxas de mortalidade - inclusive infantil - e de fecundidade. De 2002 para 2003, o número de idosos passou de 16,0 milhões (9,3\%) para 16,7 milhōes $(9,6 \%)$, sendo que as maiores proporçôes de idosos estavam no Rio de Janeiro (12,7\%) e no Rio Grande do Sul (12,5\%), estados com reduzidas taxas de mortalidade infantil, e as menores em Roraima (3,7\%) e no Amazonas (4,9\%), estados com elevadas taxas de mortalidade infantil (IBGE, 2005). Esses dados confirmam haver uma relação inversamente proporcional entre a expectativa de vida ao nascer - um indicador da existência de boa qualidade de vida - e a mortalidade infantil para o conjunto dos estados brasileiros, como verificado no presente estudo.

Holcman, Latorre \& Santos (2004) concluíram que as políticas públicas voltadas para a melhoria das condições de saneamento básico das comunidades - abastecimento de água e esgotamento sanitário - foram eficazes para diminuir a mortalidade infantil pós-neonatal, período em que os óbitos decorrem principalmente de doenças ligadas às condições do ambiente em que a criança vive. Tal achado confirma que o aumento da cobertura populacional por sistemas de esgotamento sanitário
Tabela 2 - Regressão multivariada linear entre a mortalidade por doença diarréica aguda em crianças menores de cinco anos e indicadores demográficos, sócioeconômicos, fatores de risco, gastos públicos e de cobertura por serviços

de saúde e de saneamento

\begin{tabular}{cclccc}
\hline $\mathrm{R}^{2}$ & $\mathrm{R}$ & Variáveis que permaneceram & Coef. $\beta$ & P Valor & Sinal \\
\hline 0,595 & 0,771 & Proporção de pobres & 0,575 & 0,001 & + \\
& & $\begin{array}{l}\text { Cobertura por sistemas de } \\
\text { esgotamento sanitário }\end{array}$ & $-0,324$ & 0,035 & - \\
\hline
\end{tabular}

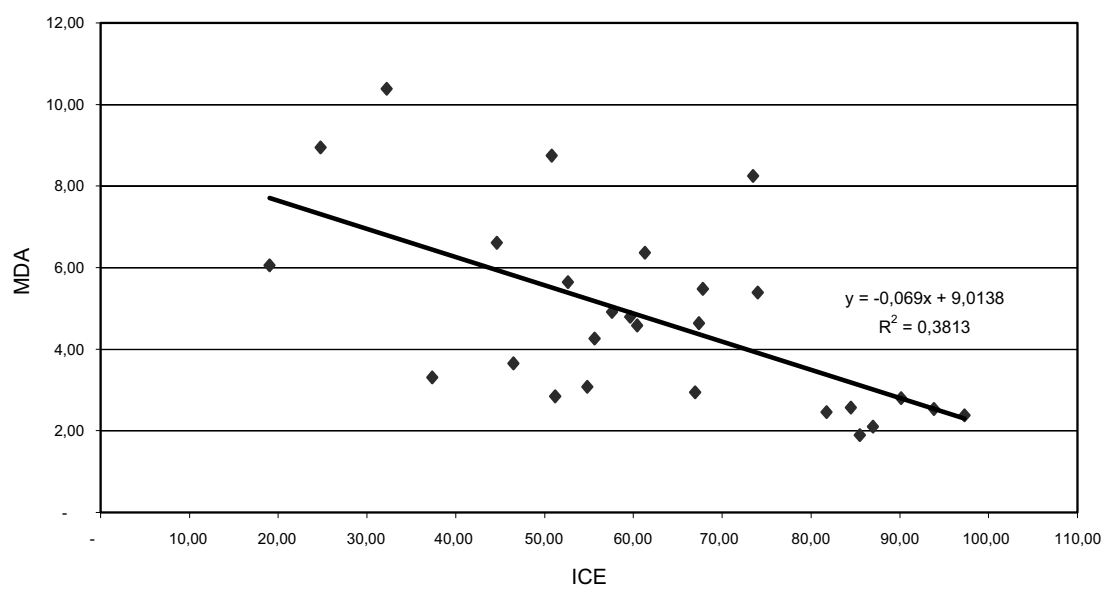

Figura 2 - Regressão linear simples entre a mortalidade proporcional por doença diarréica aguda em menores de cinco anos de idade (\% de óbitos informados) e a cobertura por sistemas de esgotamento sanitário (\% da população urbana)

Tabela 3 - Regressão multivariada linear entre a mortalidade proporcional por doenças infecciosas e parasitárias para todas as idades e indicadores demográficos, sócio-econômicos, fatores de risco, gastos públicos e de cobertura por serviços de saúde e de saneamento

\begin{tabular}{cclccc}
\hline $\mathrm{R}^{2}$ & $\mathrm{R}$ & Variáveis que permaneceram & Coef. $\beta$ & P Valor & Sinal \\
\hline 0,544 & 0,738 & $\begin{array}{l}\text { Proporção de pobres } \\
\text { Cobertura por sistemas de }\end{array}$ & 0,522 & 0,001 & + \\
& & & 0,499 & 0,001 & - \\
& esgotamento sanitário & & \\
\hline
\end{tabular}

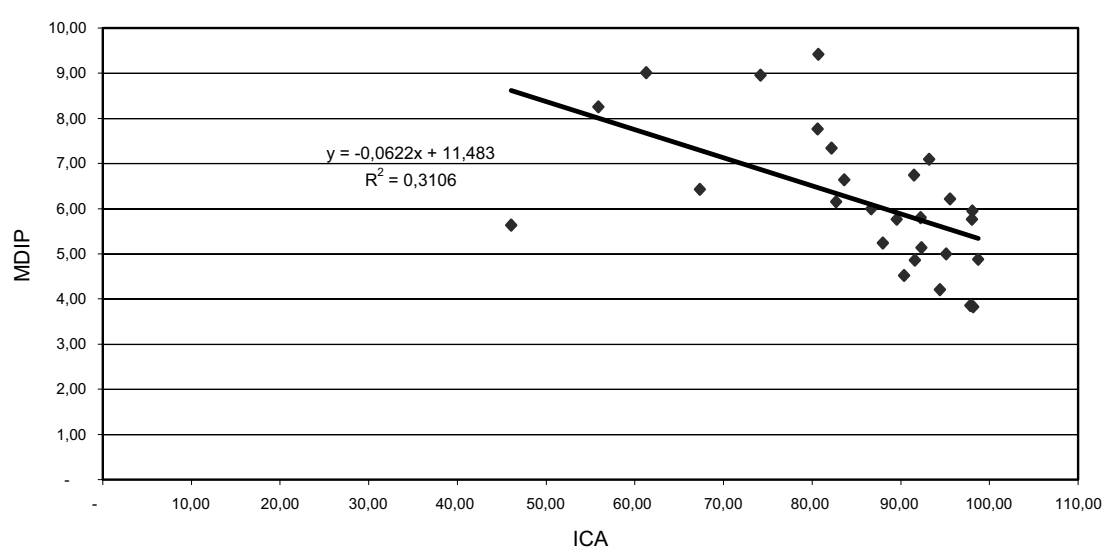

Figura 3 - Regressão linear simples entre a mortalidade proporcional por doenças infecciosas e parasitárias para todas as idades (\% de óbitos com causa definida) e a cobertura por redes de abastecimento de água (\% da população urbana) 
pode contribuir para reduzir ainda mais a mortalidade infantil no Brasil, como identificado no presente estudo.

Quanto à mortalidade proporcional por doença diarréica na infância, na cidade de São Paulo, Benicio \& Monteiro (2000) demonstraram que a ocorrência mais freqüente foi observada entre filhos de mães com pouca ou nenhuma escolaridade e entre crianças que vivem em famílias de baixa renda, confirmando o resultado deste estudo.

Corroborando achado do presente estudo, Heller, Colosimo \& Antunes (2004) destacaram, em pesquisa realizada em Betim - MG, que a incidência de diarréia é diretamente proporcional à presença de água de esgoto escorrendo pelas ruas, ou seja, pela ausência de esgotos sanitários nas comunidades.

Quanto às doenças infecciosas e parasitárias, Paes \& Silva (1999) afirmam que as DIPs atingem populações menos privilegiadas, de baixa renda, com baixo nível escolar e que não dispõem de condiçôes de saneamento básico adequados - abastecimento de água e de esgotamento sanitário - e assistência primária à saúde, confirmando os achados do presente estudo.

\section{CONCLUSÕES}

Verificou-se que o delineamento ecológico utilizado no presente estudo apresenta como principais vantagens: a facilidade de execução a partir de dados secundários, o baixíssimo custo relativo se comparado a outros métodos epidemiológicos e a simplicidade analítica. Por outro lado, as suas maiores desvantagens foram o baixo poder analítico, o pouco desenvolvimento das técnicas de análise de dados e a vulnerabilidade à falácia ecológica, ou seja, inferir conclusôes para indivíduos a partir de resultados de agregados populacionais. Em síntese, os autores corroboram as conclusões a respeito do delineamento ecológico formuladas por Almeida Filho \& Rouquayrol (2002).

$\mathrm{O}$ estudo sugeriu que a taxa de mortalidade infantil pode ser reduzida com o aumento da esperança de vida ao nascer e do percentual da população atendida por serviços de esgotamento sanitário nos estados brasileiros; que a mortalidade proporcional por diarréia aguda em menores de cinco anos de idade pode ser combatida com a redução da percentagem da população com renda familiar per capita de até meio salário mínimo e com o aumento da cobertura populacional por sistemas de esgotamento sanitário em cada estado; e, por último, que a mortalidade proporcional por doenças infecciosas e parasitárias para todas as idades pode ser diminuída com o aumento do percentual da população com abastecimento de água e com a redução da população com renda familiar per capita de até meio salário mínimo nos estados brasileiros.

Em uma análise global, observa-se que as doenças estudadas - mortalidade infantil, doença diarréica aguda em menores de cinco anos de idade e doenças infecciosas e parasitárias para todas as idades - podem ser reduzidas, entre outros fatores, por meio da ampliação da cobertura populacional por redes de abastecimento de água e por sistemas de esgotamento sanitário.

Assim, os resultados encontrados permitem afirmar que a hipótese testada se confirmou, uma vez que a ampliação da infra-estrutura sanitária em estados com precárias condições de saneamento ambiental é um investimento capaz de melhorar a condição de saúde pública existente nos estados brasileiros e, portanto, contribuir para a redução de gastos públicos e particulares com medicina curativa.

Em síntese, o trabalho mostrou que mudanças na qualidade de vida, no poder aquisitivo das famílias e a expansão dos serviços de saneamento poderão levar ao declínio da taxa de mortalidade infantil, da mortalidade proporcional por doença diarréica aguda em crianças menores de cinco anos e da mortalidade proporcional por doenças infecciosas e parasitárias para todas as idades, nos estados brasileiros.

\section{AGRADECIMENTOS}

Trabalho subvencionado pelo Programa Institucional de Bolsas de Iniciação Científica - PIBIC do Conselho Nacional de Desenvolvimento Científico e Tecnológico (Processo no 800664/1992-2).

\section{REFERÊNCIAS}

ALMEIDA FILHO, N.; ROUQUAYROL, M.Z. Introdução à epidemiologia. 3 a ed. Rio de Janeiro: MEDSI, 2002.

BENICIO, M.H. D'A.; MONTEIRO, C.A. Tendência secular da doença diarréica na infância na cidade de São Paulo (1984-1996). Revista de Saúde Pública, v.34, n.6 supl., p.83-90, 2000.

BLUM, D.; FEACHEM, R.G. Measuring the impact of water supply and sanitation investments on diarroeal diseases: problems of methodology. International Journal of Epidemiology, v.12, n.3, p.357-365, 1983.

CONFERÊNCIA DAS NAÇÕES UNIDAS SOBRE MEIO AMBIENTE E DESENVOLVIMENTO. 2ed. Brasília: Senado Federal Subsecretaria de Ediçôes Técnicas, 1997.

FIBGE - FUNDAÇÃO INSTITUTO BRASILEIRO DE GEOGRAFIA E ESTATÍSTICA. Sinopse preliminar do censo demográfico 2000. Disponível em: <http://www.ibge.gov.br/ibge/estatistica/universo.php>. Acesso: 03 fev. 2002.

FIBGE - FUNDAÇÃO INSTITUTO BRASILEIRO DE GEOGRAFIA E ESTATÍSTICA. Síntese de Indicadores Sociais 2004. Disponível em: <http://www.ibge.gov.br>. Acesso: 18 abr. 2005.

HELLER, L. Saneamento e Saúde. Brasília: OPAS/OMS, 1997.

HELLER, L.; COLOSIMO, E.A.; ANTUNES, C.M. de F. Evironmental sanitation conditions and health impact: a case-control study. Revista da Sociedade Brasileira de Medicina Tropical, v.36, n.1, p.41-50, 2003.

HOLCMAN, M.M. et al. Evolução da mortalidade infantil na região metropolitana de São Paulo 1980-2000. Revista de Saúde Pública, v.38, n.2, p.180-186, 2004.

IDB 2003-Indicadores e Dados Básicos para a Saúde 2003. Disponível em: <http://tabnet.datasus.gov.br/ cgi/idb2003/matriz.htm>. Acesso: 01 jul. 2004.

MORAES, L.R.S. Avaliação do impacto sobre a saúde das ações de saneamento ambiental em áreas pauperizadas de Salvador - Projeto AISAM. In: HELLER, L. et al. Saneamento e saúde em países em desenvolvimento. Rio de Janeiro: CC\&P Editores, p.281-305, 1997.

PAES, N.A.; SILVA, L.A.A. Doenças infecciosas e parasitárias no Brasil: uma década de transição. Revista Pan-Americana de Salud Publica, v.6, n.2, p.99-109, 1999.

ROSEN, G. Uma história da saúde pública. São Paulo: HUCITEC, (Saúde em debate; 74). 1994.

SILVA, H.K. de S.; ALVES, R.F.F. $O$ saneamento das águas no Brasil. In: O ESTADO DAS ÁGUAS NO BRASIL. BRASÍLIA: ANEEL, p. 83-102, 1999.

SNOW, J. Sobre a maneira de transmissão da cólera. 2ed. São Paulo: HUCITEC-ABRASCO, 1990.

TAVARES, R.; MONTEIRO, M.F.G. População e condições de vida. In: GUIMARÃES, R.A.; TAVARES, R. (ORGANIZADORES). SAÚDE E SOCIEDADE NO BRASIL - ANOS 80. Rio de Janeiro: Relume Dumará, 1994.

TEIXEIRA, J.C. Associação entre cenários de saneamento e indicadores de saúde em crianças. Estudo em áreas de assentamento subnormal em Juiz de Fora - MG. Belo Horizonte: Escola de Engenharia da UFMG, 2003. 287p. (Tese, Doutorado em Saneamento, Meio Ambiente e Recursos Hídricos).

Endereço para correspondência:

Júlio César Teixeira

Departamento de Hidráulica e

Saneamento

Universidade Federal de Juíz de Fora

Campus da UFJF - Martelos

36036-900 Juíz de Fora - MG - Brasil

Tel.: (32) 3232-6342

E-mail: juliotei@terra.com.br 